\title{
Detection of micrometastases in lung cancer with magnetic nanoparticles and quantum dots
}

\author{
This article was published in the following Dove Press journal: \\ International Journal of Nanomedicine \\ 4 May 2012 \\ Number of times this article has been viewed
}

\author{
Yali Wang \\ Yucheng Zhang* \\ Zhenwu Du \\ Mei Wu \\ Guizhen Zhang* \\ Central Laboratory, China-Japan \\ Union Hospital, Jilin University, \\ Changchun, People's Republic \\ of China \\ *These authors contributed equally \\ to this work
}

\begin{abstract}
Detection of micrometastases plays an important role in early-stage and recurrent cancer diagnosis. In the study, a new method of screening micrometastases of lung cancer in peripheral blood by magnetic nanoparticles (MNPs) and quantum dots (QDs) was developed to achieve early diagnosis and recurrence prevention. MNPs were prepared by combining miniemulsion polymerization and Stöber coating methods. QDs were prepared by using $\mathrm{Cd}(\mathrm{Ac})_{2} \cdot 2 \mathrm{H}_{2} \mathrm{O}$ and oxygen-free $\mathrm{NaHTe}$ with thioglycolic acid as the stabilizer. The carbodiimide-mediated condensation method was used to couple pan-cytokeratin (pan-ck) antibody $(\mathrm{Ab})$ to the surface of the MNPs, and Lunx and SP-A Abs to the surface of the QDs. After four kinds of epithelial tumor cells were enriched by MNPs coupled with pan-ck $\mathrm{Ab}$ (MNP-pan-ck), lung cancer cells A549 and SPC-A-1 were successfully identified by QDs with double-labeled Abs. Finally, 32 patients with non-small cell lung cancer (NSCLC) were collected, out of 26 cases with the enriched circulating tumor cells (CTCs), 21 cases were successfully identified by QDs. Therefore, a new method was established in which MNP-pan-ck collected CTCs and QDs with double-labeled Abs could be used simultaneously to identify CTCs from NSCLC patients.
\end{abstract}

Keywords: micrometastases, lung cancer, magnetic nanoparticles, quantum dots, Lunx, SP-A

\section{Introduction}

Lung cancer is the leading cause of cancer deaths worldwide. International standards divide lung cancer into two types: small cell lung cancer and non-small cell lung cancer (NSCLC). NSCLC accounts for approximately 85\% of all cases of lung cancer. Surgery is the preferred treatment method for NSCLC patients. However, many patients remain at risk of recurrence and metastasis following surgery, and it is difficult for routine histopathology and imageology methods to detect metastasis. Therefore, more sensitive and specific methods are needed to detect lung cancer earlier and identify patients at highest risk for recurrence. In 2002, Bernards and Weinberg ${ }^{1}$ presented a new model of metastasis where metastatic capacity is gained early during primary tumor development. Micrometastases were originally defined by pathologists as small occult metastases $(0.2 \mathrm{~cm}$ in their greatest dimension). Currently, single circulating tumor cells (CTCs) are also called micrometastases. Indeed, single CTCs in cancer patients can be detected at early stages of tumor progression in peripheral blood. In order to detect single CTCs in peripheral blood, several different methods have been developed including reverse-transcription polymerase chain reaction, flow cytometry, and the immunomagnetic beads technique..$^{2-7}$ 
To date, nanomaterials and nanotechnology have shown great potential in disease diagnosis and therapy. Magnetic nanoparticles (MNPs) are a new type of nanomaterial. ${ }^{8}$ The application of MNPs in the fields of biomedicine such as magnetic separation, drug delivery, magnetic resonance imaging, cell and tissue targeting, and transfection have drawn considerable attention due to their particularly large surface-to-volume ratio and intrinsic magnetic properties. ${ }^{9,10}$ Quantum dots (QDs) are nanocrystals of inorganic semiconductors that are restricted in three dimensions to a somewhat spherical shape, typically with a diameter of $2-8 \mathrm{~nm} .{ }^{11}$ QDs are a relatively new class of fluorescent nanomaterials with excellent photophysical properties which include quantum-size effect, high brightness, long-term photostability, high quantum yields, and more importantly, broad absorption spectra coupled to narrow size-tunable photoluminescent emission spectra. ${ }^{12}$ These properties and advantages of MNPs and QDs have provided a new functional platform for disease diagnosis and therapy.

It is well known that NSCLC is a type of epithelial cancer. Pan-cytokeratin (pan-ck) are currently the usual protein markers for the detection of epithelial tumor cells. ${ }^{13}$ Lunx, a novel human lung-specific gene, has been reported to be a superior diagnostic marker for the detection of micrometastases in peripheral blood and lymph nodes of NSCLC patients. ${ }^{14}$ Surfactant protein-A (SP-A) is the most prominent of the four proteins in the pulmonary surfactant system. Moreover, expression of SP-A was also described for a portion of NSCLC facilitating a diagnostic marker for these carcinomas. ${ }^{15,16}$

Since CTCs have very low concentration in the blood (1 per $10^{6-7}$ leukocytes), a primary enrichment step must usually be performed to augment the sensitivity of the assay. ${ }^{17}$ This will be followed by a detection method that will identify CTCs. In the present study, new modified MNPs coupled with pan-ck Ab (MNP-pan-ck) are prepared to enrich peripheral blood CTCs in NSCLC patients. After the CTCs are isolated with the MNPs, QDs conjugated with Lunx and SP-A Ab are used to identify the CTCs.

\section{Materials and methods}

\section{Preparation and modification of MNPs}

Iron oxide $\left(\mathrm{Fe}_{3} \mathrm{O}_{4}\right)$ MNPs were prepared by coprecipitation of $\mathrm{FeCl}_{2} / \mathrm{FeCl}_{3}$ (ratio 1:2) by the Massart method. ${ }^{18}$ Oleic acid (OA)-modified MNPs were produced by redispersing $\mathrm{Fe}_{3} \mathrm{O}_{4} \mathrm{MNP}$ precipitate in $150 \mathrm{~mL}$ of water and adding an excess amount of OA. ${ }^{19}$ The dark suspension was stirred for 1 hour at $70^{\circ} \mathrm{C}$ under the protection of $\mathrm{N}_{2}$. The oily black precipitate was collected, washed three times with water, and then dried in an oven under the protection of $\mathrm{N}_{2}$. Miniemulsion polymerization was used to prepare $\mathrm{Fe}_{3} \mathrm{O}_{4}$ /polystyrene MNPs according to the following procedure: ${ }^{20} \mathrm{Fe}_{3} \mathrm{O}_{4}$ MNPs were modified by $0.11 \mathrm{~g}$ of $\mathrm{OA}$ and were dispersed in a solution of $1 \mathrm{~mL}$ of styrene and $0.1 \mathrm{~g}$ of cyclohexane with the aid of ultrasound to form the oil phase. $80 \mathrm{mg}$ of sodium dodecyl sulfate (SDS) and $8 \mathrm{mg}$ sodium bicarbonate were dissolved in $30 \mathrm{~mL}$ of water to form the water phase. A mixture of the oil phase and the water phase was ultrasonicated in an ice-cooled bath for 10 minutes to form a miniemulsion and was transferred to a $250 \mathrm{~mL}$ four-necked flask equipped with a condenser, a nitrogen inlet, and a stirrer. $15 \mathrm{mg}$ of potassium persulfate dissolved in $0.5 \mathrm{~mL}$ of water was added to initiate the polymerization at $70^{\circ} \mathrm{C}$ with continual stirring at $300 \mathrm{rpm}$ (magnetic stir bars, $25 \mathrm{~mm} \times 9 \mathrm{~mm}$ ). After 12 hours, the $\mathrm{Fe}_{3} \mathrm{O}_{4}$ /polystyrene MNPs were obtained. $\mathrm{Fe}_{3} \mathrm{O}_{4}$ /polystyrene MNPs were centrifuged for 120 minutes at 21,000 rpm and dried in a desiccator for further characterization. $\mathrm{Fe}_{3} \mathrm{O}_{4} /$ polystyrene MNPs were prepared by Stöber coating methods. Carboxyl-functionalized $\mathrm{Fe}_{3} \mathrm{O}_{4} /$ polystyrene MNPs were prepared by copolymerization of acrylic acid and styrene. Transmission electron microscope (TEM) images were recorded using an H-8100 microscope (Hitachi, Tokyo, Japan).

\section{Preparation and modification of aqueous soluble CdTe QDs}

Cadmium telluride (CdTe) QDs were synthesized according to the method reported by Zhao. ${ }^{21}$ Briefly, $0.5 \mathrm{mmol}$ $\mathrm{Cd}(\mathrm{Ac})_{2} \cdot 2 \mathrm{H}_{2} \mathrm{O}$ (Beijing Chemical Int., Beijing, China) was dissolved in $100 \mathrm{~mL}$ of high-purity water, then $87 \mu \mathrm{L}$ of thioglycolic acid (Acros, Geel, Belgium) was added into the mixture under stirring. $1 \mathrm{M} \mathrm{NaOH}$ was used to adjust the $\mathrm{pH}$ of the solution to 9.5. After the mixture had been degassed by high-purity $\mathrm{N}_{2}$ bubbling for 30 minutes, a $0.25 \mathrm{mmol}$ oxygen-free NaHTe (Beijing Chemical Int.) solution prepared by reducing Te powder by $\mathrm{NaBH}_{4}$ was injected. The resulting mixture was subjected to reflux until the appreciated size was reached by monitoring the ultraviolet-visible curves of the aliquot. Fluorescence spectra of CdTe QDs were measured on a FS900 fluorescence spectrometer (Edinburgh Analytical Instruments, Edinburgh, UK). High-resolution transmission electron microscope (HRTEM) characterizations were performed by using a JSM-3010 (JEOL, Tokyo, Japan) electron microscope. In the experiment, two kinds of CdTe QDs were synthesized, and were named QD552 and QD625, respectively, based on the maximum fluorescence emission of the QDs. 


\section{Coupling pan-ck $A b$ to the surface of MNPs}

The carbodiimide-mediated condensation method was used to couple pan-ck Ab to the surface of MNPs. Briefly, $100 \mu \mathrm{L}$ of MNPs were transferred to a reaction tube and coupling buffer (10 mM K $\mathrm{HPO}_{4}, 150 \mathrm{mM} \mathrm{NaCl}, \mathrm{pH}$ 5.5) was added to a final volume of $1 \mathrm{~mL}$. The reaction tube was shaken vigorously for 1 minute and then the MNPs were separated magnetically by placing the reaction tube in the magnetic separator (Dynal AS, Oslo, Norway) at $4^{\circ} \mathrm{C}$ for 5 minutes. The supernatant was removed, leaving the MNPs as a wet cake on the container wall. The MNPs were suspended in $1 \mathrm{~mL}$ of coupling buffer. $0.25 \mathrm{mg}$ 1-ethyl-3-(3-dimethylaminopropyl) carbodiimide (EDAC) reagent (Sigma-Aldrich, St Louis, MO) was added to the system and stirred briefly. $50 \mu \mathrm{g}$ pan-ck $\mathrm{Ab}$ was added to the reaction system and vortexed for 1 hour. The MNP-pan-ck was magnetically separated and the supernatant was removed. $1 \mathrm{~mL}$ of wash buffer $(10 \mathrm{mM}$ Tris, $150 \mathrm{mM} \mathrm{NaCl}, 0.1 \% \mathrm{BSA}, 0.1 \% \mathrm{NaN}_{3}, 1 \mathrm{mM}$ EDTA, $\mathrm{pH}$ 7.4) was added and shaken vigorously. The wash step was repeated three times. Finally, the MNP-pan-ck was resuspended in the wash buffer.

\section{Coupling Abs to the surface of CdTe QDs}

The resultant CdTe QDs were conjugated to different lung tissue-specific Abs. QD552 was conjugated to rabbit antihuman Lunx Ab (QD552-Lunx) and QD625 was conjugated to rabbit anti-human SP-A Ab (QD625-SP-A) (Santa Cruz Biotechnology, Santa Cruz, CA). Briefly, $100 \mu \mathrm{L}$ of $1 \mathrm{mM}$ CdTe QD solution was mixed with $400 \mu \mathrm{L}$ of $0.05 \mathrm{M}$ borate buffer ( $\mathrm{pH}$ 7.5). Then, $10 \mu \mathrm{L}$ of $25 \mathrm{mg} / \mathrm{mL}$ EDAC freshly dissolved in water was added to a mixture. After incubation for 5 minutes, $20 \mu \mathrm{g}$ Ab was added to the mixture. The sample was dialyzed against phosphate-buffered saline (PBS; $10 \mathrm{mM}, \mathrm{pH}$ 7.4) after incubation in the dark for 2 hours at room temperature. When the dialysis was over, the QDs conjugated to Abs were collected.

\section{Enriching of epithelial cancer cells by MNPs}

Lung cancer A549, SPC-A-1, liver cancer HepG2, and colon cancer HCT-8 cell lines were obtained from American Type Culture Collection (Manassas, VA). All cells were maintained in Dulbecco's modified Eagle's medium (Gibco, Grand Island, NY) supplemented with $10 \%$ fetal bovine serum and were grown in $5 \% \mathrm{CO}_{2}$, at $37^{\circ} \mathrm{C}$. Diverse proportion cancer cells were mixed with peripheral blood mononuclear cells (PBMC) to achieve cell suspension (the ratios of cancer cells to PBMC of 1:10 $10^{4}, 1: 5 \times 10^{4}, 1: 10^{5}, 1: 5 \times 10^{5}, 1: 10^{6}, 1: 5 \times 10^{6}$, and $\left.1: 10^{7}\right)$. MNPs-pan-ck were added to the cell suspension and mixed gently for 15 minutes. Cancer cells originating from epithelial tumors were magnetically separated and the isolated cells were resuspended using PBS buffer, and then the separated cells were smeared onto a slide. The slides were stained with hematoxylin and eosin (H\&E) to identify cancer cells.

\section{Identification of lung cancer cells by immunocytochemistry}

A549, SPC-A-1, HepG2, and HCT-8 cells were fixed with a mixture of glacial acetic acid:ethanol (volume ratio of 1:9) for 15 minutes and air-dried for 30 minutes. Fixed cancer cells were penetrated with $0.3 \%$ Triton $\mathrm{X}-100$ for 10 minutes. The cells were respectively blocked with $\mathrm{H}_{2} \mathrm{O}_{2}$ reagent and normal goat serum for 10 minutes followed by incubating the slides with $1 \mu \mathrm{g} / \mathrm{mL}$ rabbit anti-human Lunx or SP-A Ab at $4^{\circ} \mathrm{C}$ overnight. $1.5 \mu \mathrm{g} / \mathrm{mL}$ biotinylated goat anti-rabbit IgG was incubated for 30 minutes, and then streptavidin-conjugated horseradish peroxidase was incubated for 30 minutes. Staining was achieved by adding $100 \mu \mathrm{L}$ of 3,3'diaminobenzidine for 5 minutes. Finally, slides were washed three times with PBS buffer, the nuclei were counterstained with $\mathrm{H} \& \mathrm{E}$, and then slides were rinsed with water and dehydrated in $70 \%, 90 \%$, and $100 \%$ ethanol, air-dried, and mounted with gum and viewed under an ordinary optical microscope.

\section{Identifying lung cancer cells with double-labeled Lunx and SP-A Abs QDs}

After using MNP-pan-ck to enrich the epithelial cancer cells, all cells were identified by using QD552-Lunx and QD625SP-A Abs QDs. Briefly, enriched cells were incubated with QD552-Lunx and QD625-SP-A Abs at $4^{\circ} \mathrm{C}$ overnight. Slides were washed three times with PBS buffer, mounted with glycerin reagent, and viewed under fluorescent microscope (Olympus, Tokyo, Japan).

\section{Clinical specimens}

Thirty-two patients with primary NSCLC were enrolled between May 2011 and October 2011 at China-Japan Union Hospital, including 16 males and 16 females aged between 45-77 years old. Thirty-two patients were confirmed to have NSCLC after surgical inspection and were diagnosed without history of other epithelial tumors. NSCLC patients were staged using the tumor-node-metastasis system, including 
seven cases of stage I and 25 cases of stage II-IV. Moreover, 16 patients with benign lung diseases (BLD) and 16 healthy volunteers were enrolled as controls. $10 \mathrm{~mL}$ of blood was collected from all participants and PBMC were isolated by density gradient centrifugation. CTCs were enriched from PBMC by MNP-pan-ck and identified by QDs with doublelabeled Abs. The study was approved by the Institutional Review Board of the University Hospital. All samples were collected after obtaining written informed consent.

\section{Statistical analysis}

All the data were analyzed with SPSS software (v 12.0; SPSS Inc, Chicago, IL). Experiments were performed in triplicate. Results were expressed as mean \pm standard error of mean. Statistical significance was set as $P<0.05$.

\section{Results and discussion Characterization of MNPs}

Many approaches have been employed to prepare MNPs including conventional emulsion polymerization, ${ }^{22}$ soapless emulsion polymerization, ${ }^{23,24}$ inverse emulsion polymerization, ${ }^{25}$ miniemulsion polymerization, ${ }^{26}$ and atom transfer radical polymerization. ${ }^{27,28}$ Among them, miniemulsion polymerization has been considered as one of the most advantageous encapsulation methods. In the present study, the MNPs with defined structure and controllable magnetite content were successfully prepared from monolayer OAmodified $\mathrm{Fe}_{3} \mathrm{O}_{4}$ nanoparticles through miniemulsion polymerization. Figure 1 illustrates the TEM images of different synthetic phases of the MNPs. Figure 1A shows the $\mathrm{Fe}_{3} \mathrm{O}_{4}$ nanoparticles. The diameter of the $\mathrm{Fe}_{3} \mathrm{O}_{4}$ nanoparticles is in the range from 6 to $15 \mathrm{~nm}$ with an average size of $9.6 \mathrm{~nm}$. Despite the OA-modified $\mathrm{Fe}_{3} \mathrm{O}_{4}$ nanoparticles, the mean diameter of the $\mathrm{Fe}_{3} \mathrm{O}_{4}$ nanoparticles is also $9 \mathrm{~nm}$ (Figure 1B), which implies that OA modification does not change the diameter of the $\mathrm{Fe}_{3} \mathrm{O}_{4}$ nanoparticles. Figure $1 \mathrm{C}$ shows the $\mathrm{Fe}_{3} \mathrm{O}_{4} /$ polystyrene MNPs. The results demonstrate that there are no pure polystyrene particles and bare magnetite particles without polystyrene coating. Figure 1D shows the carboxylfunctionalized $\mathrm{Fe}_{3} \mathrm{O}_{4}$ /polystyrene MNPs. A polyacrylic acid layer surfaces the $\mathrm{Fe}_{3} \mathrm{O}_{4}$ /polystyrene MNPs, which gives a fine hydrophily and biocompatibility to the MNPs.

\section{Characterization of CdTe QDs}

Several methods have been developed to synthesize watersoluble QDs for use in biologically relevant studies. ${ }^{29,30}$ In the present experiment, two kinds of CdTe QDs were synthesized (Figure 2). In Figure 2A, two kinds of QDs emit
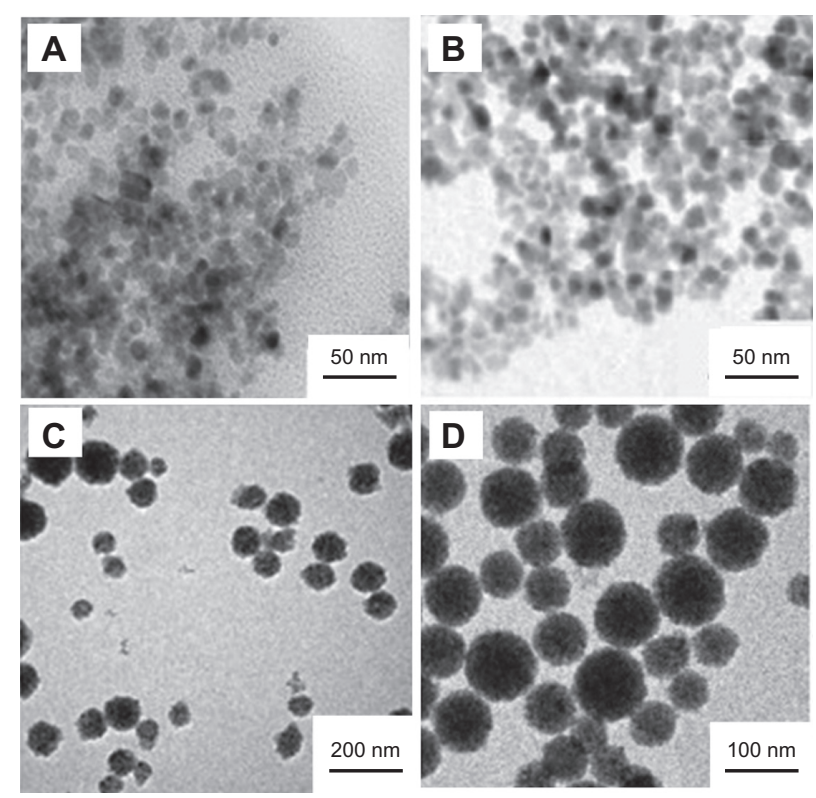

Figure I TEM images of different synthetic phases of the MNPs. (A) $\mathrm{Fe}_{3} \mathrm{O}_{4}$ nanoparticles. (B) OA-modified $\mathrm{Fe}_{3} \mathrm{O}_{4}$ nanoparticles. (C) $\mathrm{Fe}_{3} \mathrm{O}_{4} /$ polystyrene MNPs. (D) Carboxyl-functionalized $\mathrm{Fe}_{3} \mathrm{O}_{4}$ /polystyrene MNPs.

Abbreviations: OA, oleic acid; MNPs, magnetic nanoparticles; TEM, transmission electron microscopy.

different colors under UV radiation: one emits green light and the other emits red light. The HRTEM study illustrates that the diameter of CdTe QDs ranges from $2.8 \mathrm{~nm}$ to $7.3 \mathrm{~nm}$ with an average size of $4 \mathrm{~nm}$, which suggests that the CdTe QDs exhibit a good dispersion of particles, and no obvious QD aggregation can be found (Figure 2B). The fluorescence emission spectra of the CdTe QDs are shown in Figure 2C. The maximum fluorescence emission of two kinds of QDs are respectively $552 \mathrm{~nm}$ and $625 \mathrm{~nm}$, which were named after QD552 and QD625 in this study. The average full width at half the maximum of the photoluminescence spectra is about $50 \mathrm{~nm}$, which shows that the monodispersity of the QDs is quite good. The two kinds of QDs have a broader absorption spectra which show that a single light source can be used to excite two-color QDs simultaneously without signal overlap (Figure 2D). Therefore, due to these properties, the synthesized QDs provided a powerful foundation for further applications.

\section{MNP-pan-ck enriched epithelial tumor cells}

Because pan-ck Ab usually can be used to increase the sensitivity of epithelial tumor cells detection, the study chose MNP-pan-ck to enrich the CTCs in NSCLC patients. In the experiment, it was first verified whether four kinds of epithelial tumor cell lines expressed pan-ck protein by 
A

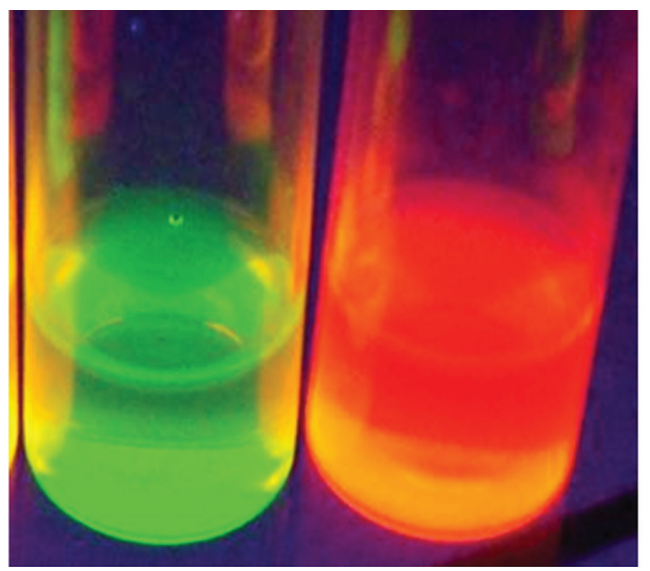

C

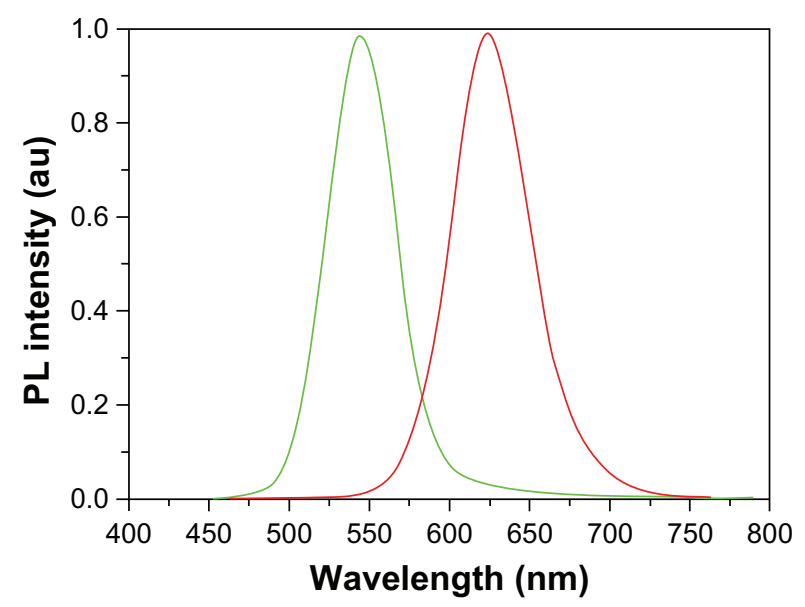

B

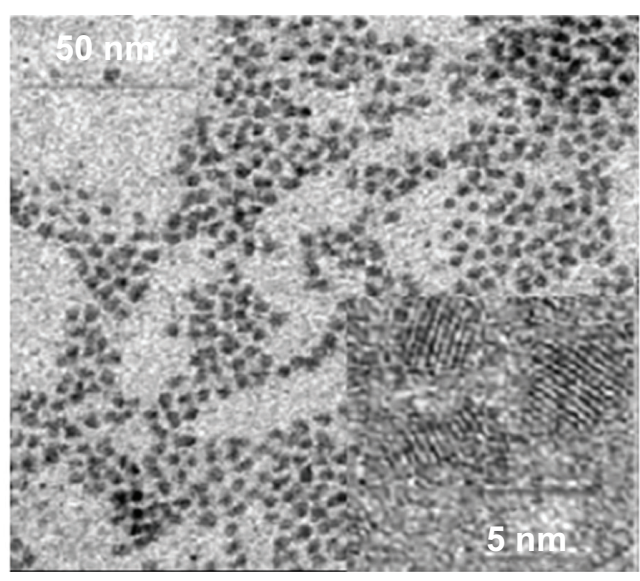

D

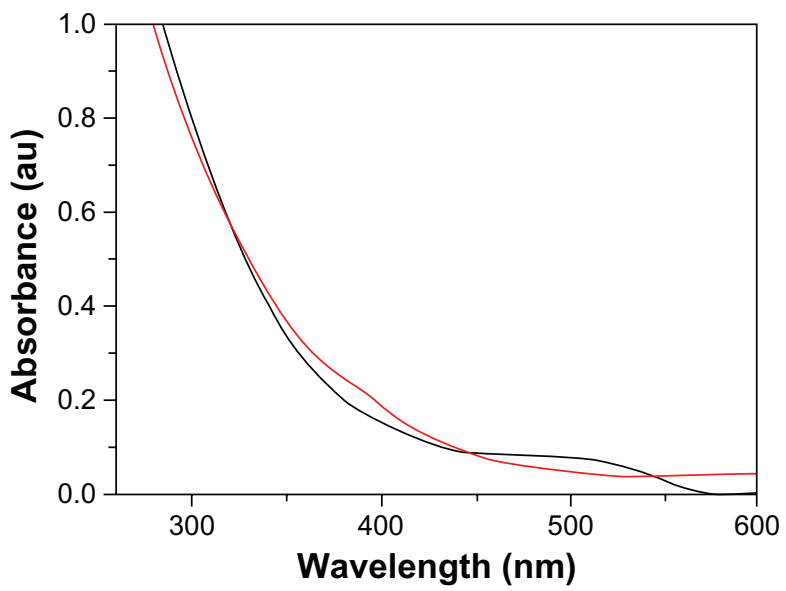

Figure 2 Properties of CdTe QDs. (A) Images of two kinds of CdTe QDs solution under ultraviolet light. (B) HRTEM images of CdTe QDs. (C) Photoluminescence spectra of two kinds of QDs. (D) Absorption spectrum of two kinds of CdTe QDs.

Abbreviations: HRTEM, high-resolution transmission electron microscope; QDs, quantum dots; CdTe, cadmium telluride; au, arbitrary units.

using the immunocytochemistry (ICC) method. The results show that the four kinds of epithelial tumor cells express pan-ck protein (Figure 3), which proves that pan-ck might be a protein probe for the enrichment of epithelial tumor cells. Secondly, in order to mimic an in vivo environment, the four kinds of epithelial tumor cell lines were mixed with PBMC in different proportions in vitro. Then, MNP-pan-ck was used to enrich the four kinds of epithelial tumor cells. With a ratio of cancer cells to PBMC of 1:5 $\times 10^{6}$, the tumor cells were successfully enriched. Furthermore, the enriching of cancer cells was increased by elevating the proportion of cancer cells to PBMC (Figure 4). After enrichment, the H\&E-stained cancer cells demonstrate that the four kinds of epithelial tumor cells are successfully separated from the mixture of cancer cells and PBMC. However, no cells from PBMC were enriched (Figure 5).

Collectively, in order to mimic an in vivo environment, different kinds of epithelial tumor cells were isolated from the mixture of PBMC and cancer cells by using MNP-pan-ck in vitro. Therefore, it was thought that MNP-pan-ck might separate CTCs in the peripheral blood of NSCLC patients.

\section{Expression of Lunx and SP-A in different cancer cells by ICC and QD methods}

Because A549 and SPC-A-1 cells are derived from NSCLC, and Lunx and SP-A protein belong to lung tissue-specific proteins, it is hypothesized that A549 and SPC-A-1 lung cancer cells might also express Lunx and SP-A proteins, while liver cancer HepG2 and colon cancer HCT-8 cells do not express both proteins. To verify expression of Lunx and SP-A protein in the four kinds of cancer cells, QDs conjugated with Lunx and SP-A Ab were combined with the ICC method for detection purposes.

In our study, the traditional ICC method was used first to detect the expression of Lunx and SP-A protein in different cancer cells. We found that both A549 and SPC-A-1 cells 


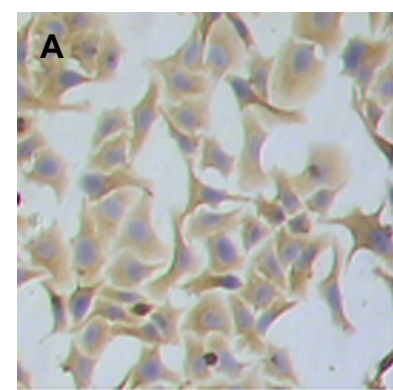

A549

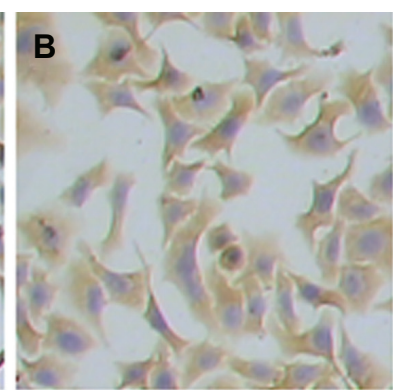

SPC-A-1

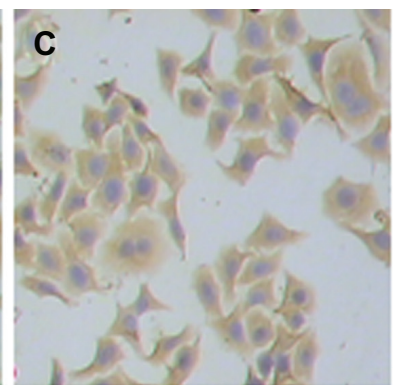

HepG2

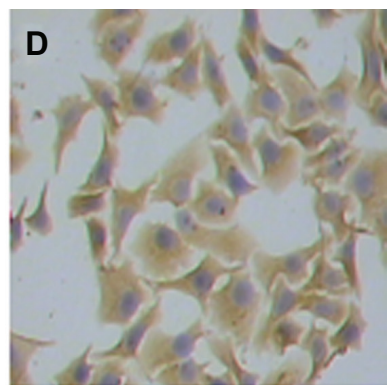

HCT-8

Figure 3 The images of pan-ck protein expression in four kinds of epithelial tumor cells by the ICC method. (A) Lung cancer A549 cells. (B) Lung cancer SPC-A-I cells. (C) Liver cancer HepG2 cells. (D) Colon cancer HCT-8 cells.

Note: The magnification was $100 \times$.

Abbreviations: ICC, immunocytochemistry; pan-ck, pan-cytokeratin.

express Lunx protein (Figure 6A and B) and positive cell rates are $57.43 \% \pm 2.47 \%$ and $48.26 \% \pm 3.42 \%$, respectively, while HepG2 and HCT- 8 cells do not express Lunx protein (Figure 6C and D). At the same time, the results also show that only A549 cells express SP-A protein (Figure 6E) and its positive cell rate was $36.25 \% \pm 2.66 \%$, while SPC-A- 1 , HepG2, and HCT-8 cancer cell lines do not express SP-A protein (Figure $6 \mathrm{~F}-\mathrm{H}$ ). Therefore, the results prove that Lunx and SP-A protein might be lung tissue-specific proteins and important markers of lung cancer cells.

Next, in order to assess whether QDs conjuncted with Abs showed similar results with the ICC method, QDs with singlelabeled $\mathrm{Ab}$ were used to detect the expression of Lunx and
SP-A protein in four kinds of cancer cells. After four kinds of cancer cells were single-labeled with QD552-Lunx or QD625SP-A, both A549 and SPC-A-1 lung cancer cells emit green fluorescence, which indicates that two kinds of lung cancer cells express Lunx protein (Figure 7A and B), and positive cell rates were $58.37 \% \pm 2.54 \%$ and $50.33 \% \pm 2.97 \%$, respectively. On the other hand, A549 cells only emit red fluorescence (Figure 7E), which indicates that only A549 cells express SP-A protein and its positive cell rate is $38.28 \% \pm 3.13 \%$. Furthermore, neither HepG2 nor HCT- 8 cells emit any fluorescence signal, which implies that these cells do not express Lunx and SP-A protein. There is no significant difference in the results between the ICC method and the method of QDs

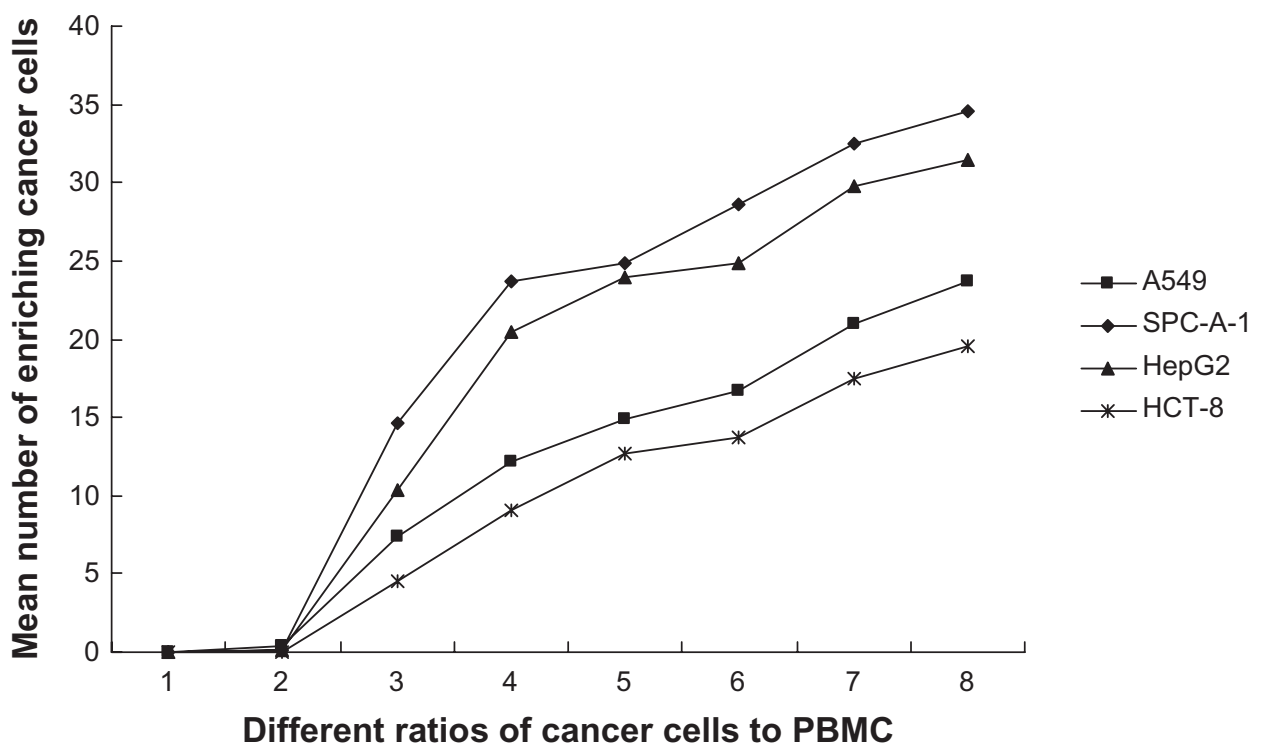

Figure 4 The mean number of enriching cancer cells from the different ratios of cancer cells to PBMC by MNP-pan-ck.

Notes: When the ratio of cancer cells to PBMC is $1: 5 \times 10^{6}$ (Group 3), the cancer cells are successfully enriched by MNP-pan-ck, and when the ratios of cancer cells to PBMC increases, the ability to enrich cancer cells is increased. The ratios of cancer cells to PBMC: Group I, only PBMC; Group 2, I:107; Group 3, I:5 × 106; Group 4, I:106; Group 5, I:5 × 105; Group 6, 1:105; Group 7, 1:5 × 104; Group 8, 1:104.

Abbreviations: PBMC, peripheral blood mononuclear cells; MNP-pan-ck, MNPs coupled with pan-cytokeratin antibody. 


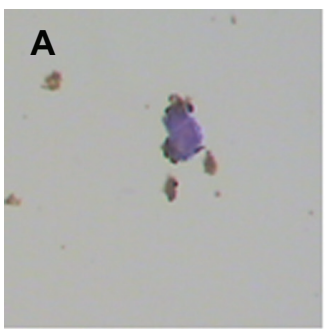

A549

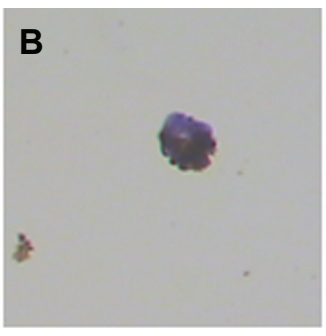

SPC-A-1

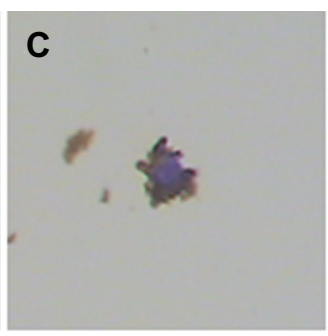

HepG2

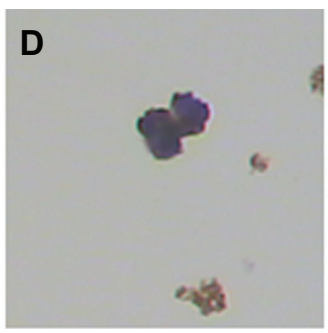

HCT-8

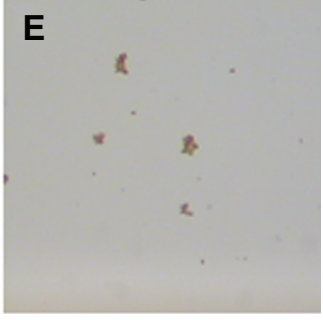

PBMC

Figure 5 H\&E-stained cancer cells enriched by MNPs. (A) A549 cells. (B) SPC-A-I cells. (C) HepG2 cells. (D) HCT-8 cells. (E) No cells are enriched in the PBMC group. Note: The magnification was $100 \times$.

Abbreviations: H\&E, hematoxylin and eosin; MNPs, magnetic nanoparticles; MNP-pan-ck, MNPs coupled with pan-cytokeratin antibody.

with single-labeled $\mathrm{Ab}(P>0.05)$. Therefore, the results of QDs with single-labeled Ab are consistent with the ICC method, which prove that QDs with labeled Ab can be used to detect different tumor cells.

QDs show many excellent photophysical properties. One of the most important is that they have broad absorption spectra and narrow size-tunable photoluminescent emission spectra. Thus, it is hypothesized that QDs with different Abs can be used to simultaneously check different specific markers of tumors. In order to validate this hypothesis, a combination of QD552-Lunx and QD625-SP-A was used to check the above four cancer cells in vitro. As shown in Figure 7I,
A549 cells emit not only red fluorescence, but also green fluorescence, and the positive cell rates are $75.78 \% \pm 3.21 \%$, while the results of SPC-A-1, HepG2, and HCT-8 cells are the same as that of QDs with single-labeled Ab. Therefore, the study demonstrates that QDs with double-labeled Abs have more sensitivity than QDs with single-labeled Ab while using the ICC method.

Finally, in order to mimic an in vivo environment, QDs with double-labeled Abs were used to identify four kinds of cancer cells that had been separated from the mixture of cancer cells and PBMC by MNP-pan-ck. Figure 8 illustrates that A549 and SPC-A-1 cells are successfully identified by
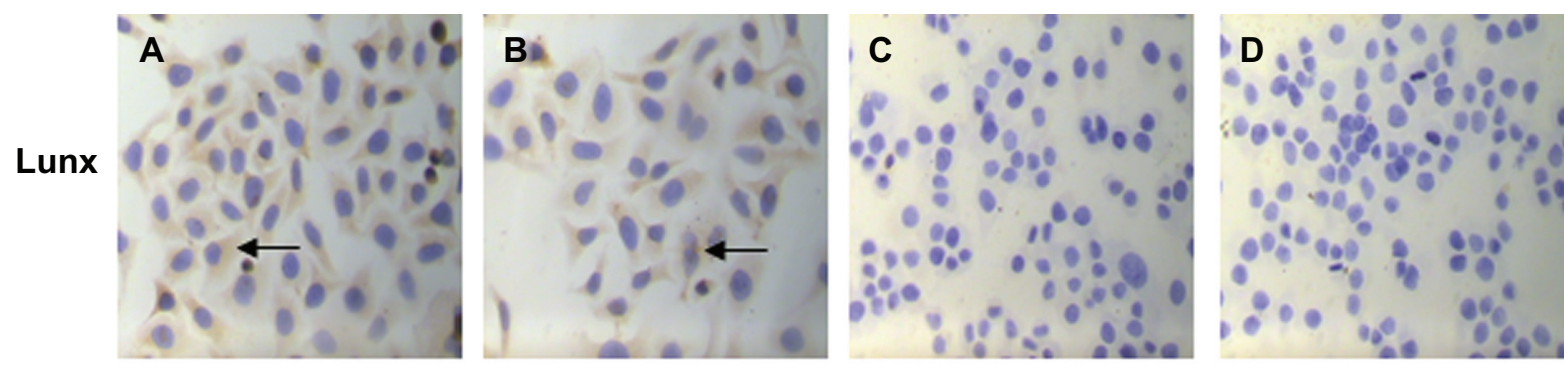

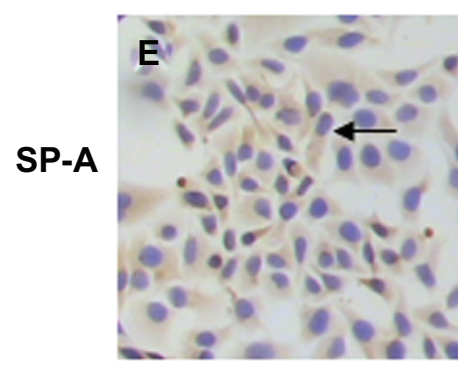

A549

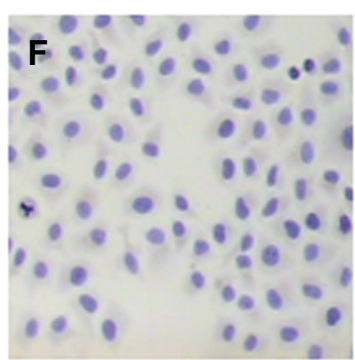

SPC-A-1

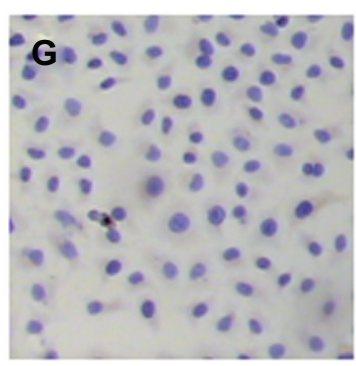

HepG2

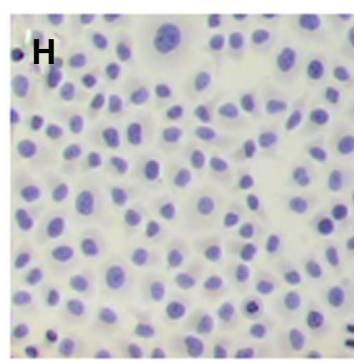

HCT-8

Figure 6 Imaging Lunx and SP-A protein expression in different cancer cells with the ICC method. Following ICC with anti-human Lunx or SP-A Ab, the results were observed in the cytoplasm of different kinds of cancer cells. The brown-colored deposits (arrows) located in the cytoplasm of cancer cells indicates that the cancer cells express the protein. The magnification was 100x. Lunx protein can be expressed in A549 (A) and SPC-A-I lung cancer cells (B). Lunx protein can not be expressed in HepG2 (C) and HCT-8 cancer cells (D). SP-A protein can be expressed only in A549 lung cancer cells (E). SP-A protein can not be expressed in SPC-A-I lung cancer cells (F), HepG2 (G) and HCT-8 cells $(\mathbf{H})$.

Abbreviations: ICC, immunocytochemistry; SP-A, surfactant protein-A; Ab, antibody. 


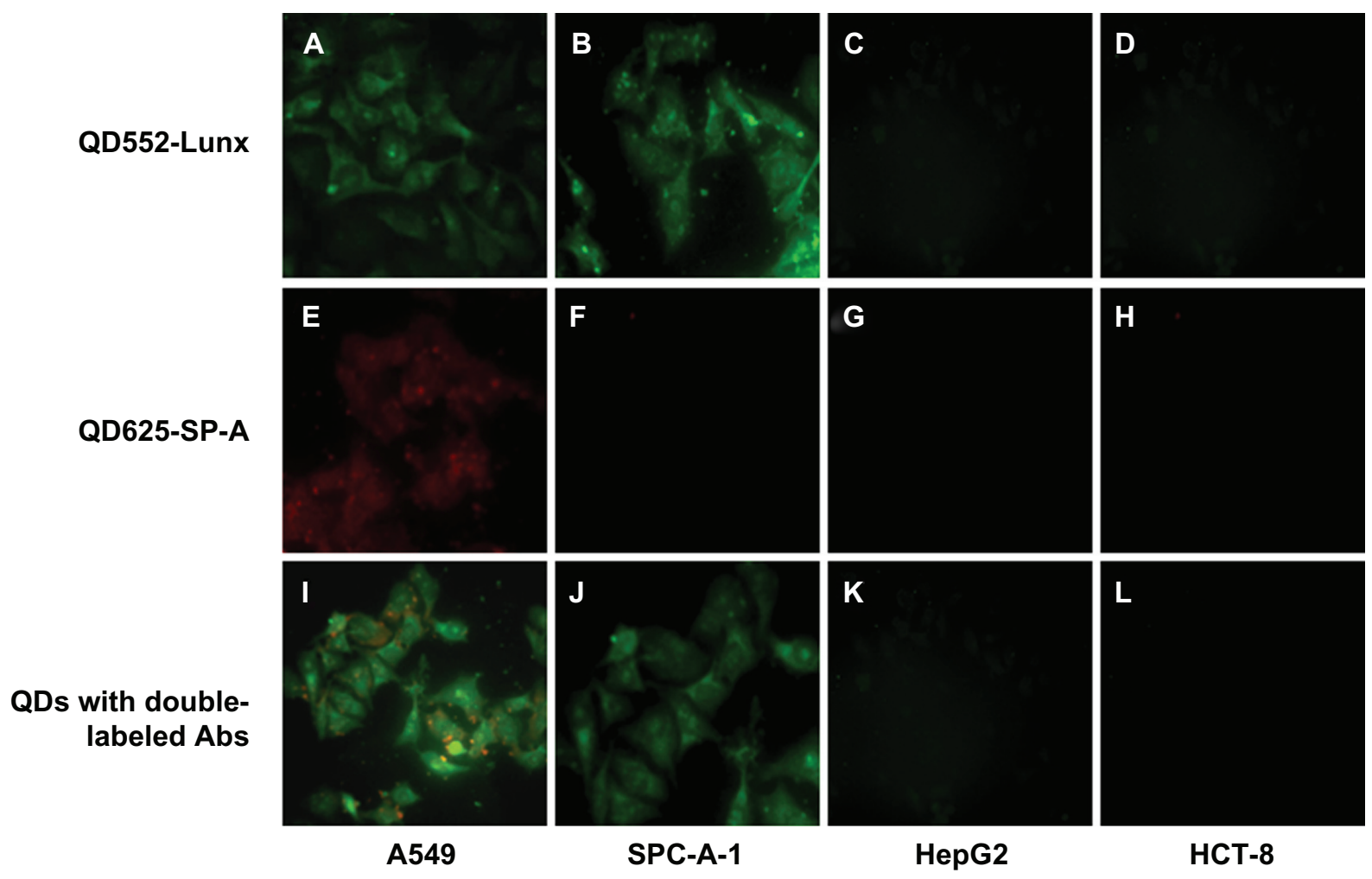

Figure 7 QDs with labeled Abs show Lunx and SP-A protein expression in different cancer cells. A549 cells (A) and SPC-A-I cells (B) labeled with QD552-Lunx show green fluorescence. A549 cells (E) labeled with QD625-SP-A show red fluorescence and SPC-A-I cells (F) labeled with QD625-SP-A do not show red fluorescence. A549 cells labeled with both QD552-Lunx and QD625-SP-A show both green and red fluorescence (I). SPC-A-I cells labeled with both QD552-Lunx and QD625-SP-A show green fluorescence (J). Neither HepG2 nor HCT-8 cells emit any fluorescence signal (C, D, G, H, K and L).

Note: The magnification was 100x.

Abbreviations: Abs, antibodies; SP-A, surfactant protein-A; QDs, quantum dots.

using QDs with double-labeled Abs (Figure 8A and B). Because both HepG 2 cells and HCT- 8 cells did not express Lunx and SP-A proteins, no fluorescence signal was observed (Figure 8C and D).

Collectively, the expressions of Lunx and SP-A proteins were first verified by using the ICC method and QDs with single-labeled $\mathrm{Ab}$. The study shows that the results of the two methods are very similar; A549 cells express both Lunx and SP-A proteins and SPC-A-1 cells only express the Lunx protein, while HepG2 and HCT-8 cells do not express either of them. Then, QDs with double-labeled Abs were used to check four kinds of cancer cells. It was found that this method could greatly improve the sensitivity of detection. Finally, QDs with double-labeled Abs identified lung cancer cells isolated by MNP-pan-ck. Therefore, this experiment found a new method for MNPs separation and QDs with double-labeled

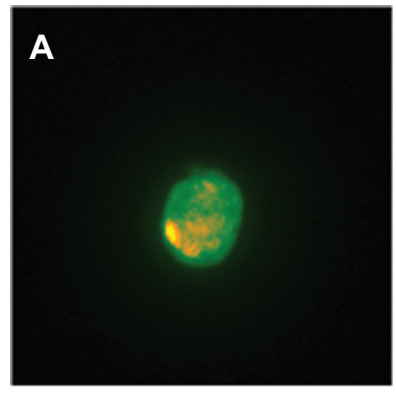

A549

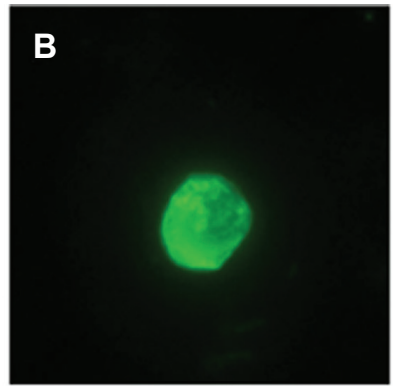

SPC-A-1

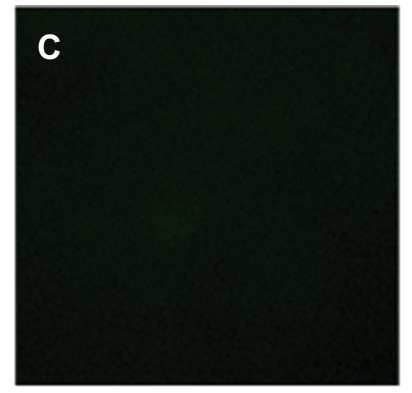

HepG2

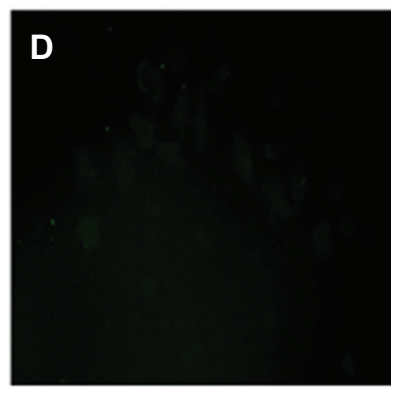

HCT-8

Figure 8 QDs with double-labeled Abs were used to identify the four kinds of enriched cancer cells. (A) A549 cells show green and red fluorescence. (B) SPC-A-I cells show green fluorescence. HepG2 cells (C) and HCT-8 cells (D) do not express Lunx and SP-A proteins, no fluorescence signal was observed.

Note: The magnification was $200 x$

Abbreviations: Abs, antibodies; MNP-pan-ck, MNPs coupled with pan-cytokeratin antibody; PBMC, peripheral blood mononuclear cell; QDs, quantum dots. 
Abs identified lung cancer cells from a mixture of cancer cells and PBMC in vitro.

\section{Detection of CTCs from NSCLC patients by MNPs and QDs}

After establishment of the method for enriching and identification of lung cancer cells in vitro, the authors wanted to verify if the in vitro method could be used to isolate and identify CTCs in peripheral blood of NSCLC patients. Therefore, the blood from 32 patients with primary NSCLC, 16 patients with BLD, and 16 healthy volunteers was collected. CTCs of 26 cases in 32 NSCLC patients were collected by MNP-pan-ck (Table 1). Of 26 cases with the enriched CTCs, 21 cases were successfully identified by QDs with double-labeled Abs, including two cases out of seven Stage I and 19 cases out of 23 Stage II-IV NSCLC patients. The positive rate of identification was $80.77 \%$, which showed that the method had a high sensitivity of isolation and identification of CTCs. No CTCs were obtained from the blood of 16 healthy volunteers and BLD using MNP-pan-ck, indicating a specificity of $100 \%$. The results suggest that a new method had been successfully found: MNP-pan-ck collects CTCs in NSCLC patients and QDs with double-labeled Abs can be used simultaneously to identify CTCs in NSCLC patients. Therefore, the method might contribute to the discovery of early micrometastases, prognosis evaluation, monitoring of recurrence, and preparation of individual postoperative treatments about NSCLC patients. However, further clinical trials may be warranted to assess the practicality of the method.

Based on the above studies, Figure 9 schematically illustrates the processes by which the MNP-pan-ck enrich the CTCs from NSCLC patients and QDs with

Table I The isolated and identified results of different groups

\begin{tabular}{llll}
\hline Group & N & MNPs enrichment (\%) & QDs identification (\%) \\
\hline NSCLC & 32 & $26(8 I .25 \%)$ & $21(80.77 \%)$ \\
Stage I & 7 & $3(42.86 \%)$ & $2(66.67 \%)$ \\
Stage II-IV & 25 & $23(92 \%)$ & $19(82.61 \%)$ \\
BLD & 16 & 0 & 0 \\
Volunteers & 16 & 0 & 0 \\
\hline
\end{tabular}

Abbreviations: MNPs, magnetic nanoparticles; QDs, quantum dots; NSCLC, nonsmall cell lung cancer; BLD, benign lung diseases.

Note: In NSCLC, stage I means that the cancer is only in the lung, with normal tissue around it. Stage II means that the cancer has spread to nearby lymph nodes. Stage III means that the cancer has spread to the chest wall or diaphragm near the lung; to the lymph nodes in the area separating the two lungs (the mediastinum); or to the lymph nodes on the other side of the chest or in the neck. Stage IV means that the cancer has spread to other parts of the body. Collectively, stages II-IV mean that the cancer has metastasized.
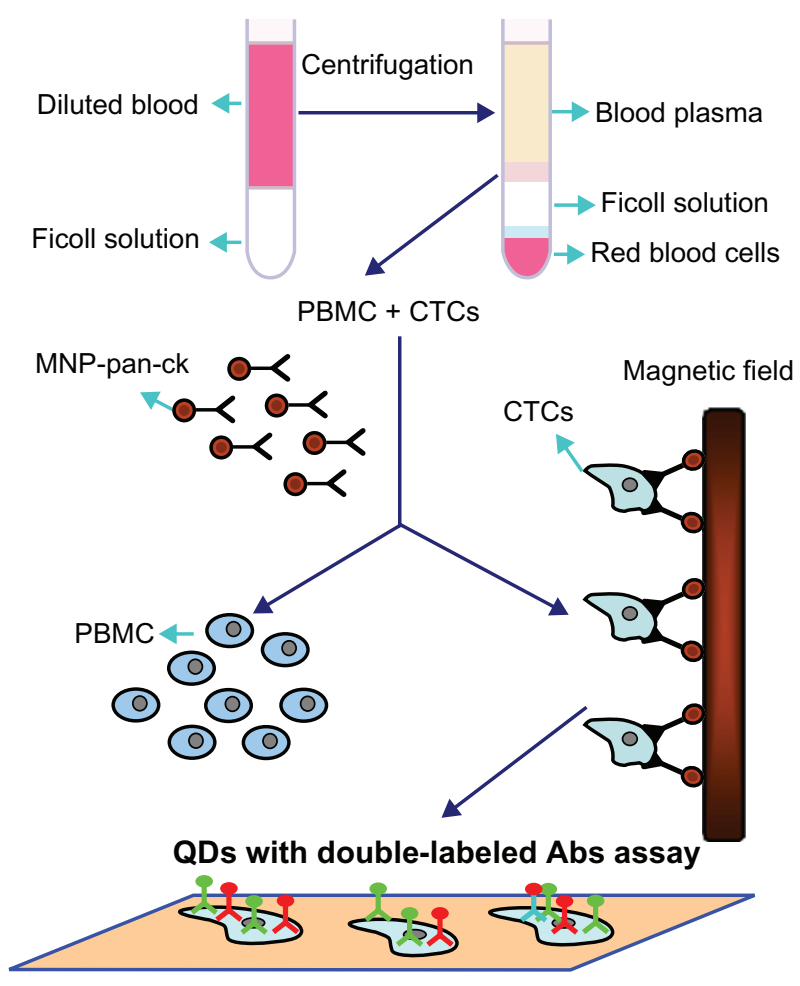

Figure 9 Schematic illustration of the isolation and identification of CTCs with MNP-pan-ck and QDs with double-labeled antibody.

Abbreviations: Abs, antibodies; CTCs, circulating tumor cells; MNP-pan-ck, MNPs coupled with pan-cytokeratin antibody; PBMC, peripheral blood mononuclear cell; QDs, quantum dots.

double-labeled Abs are used simultaneously to identify CTCs. Firstly, peripheral blood of different clinical specimens was taken, and then PBMC and CTCs were successfully separated by density gradient centrifugation. After MNP-pan-ck was used to enrich the CTCs from the specimens, QDs with double-labeled Abs were used simultaneously to identify the CTCs.

\section{Conclusion}

In this study, the synthesized MNPs were effectively coupled with pan-ck Ab, and two kinds of synthesized QDs were conjugated with Lunx and SP-A Abs. QDs with double-labeled Abs were first used simultaneously to identify the CTCs of NSCLC patients collected by MNP-pan-ck. Therefore, a new method of screening micrometastases of lung cancer in peripheral blood by MNPs and QDs was successfully developed.

\section{Acknowledgments}

This study was supported by a grant from the Science and Technology (No 20082123) of Jilin Province, China. The authors are grateful to the Key Laboratory of Surface and Interface Chemistry of Jilin University for the kind provision 
of the method of preparation and modification of $\mathrm{Fe}_{3} \mathrm{O}_{4} /$ polystyrene MNPs (patent delegation NO CN100425627C) and aqueous soluble CdTe QDs, and to Dr Wei Zhang (Chinese Academy of Medical Sciences) for providing the pan-ck Ab.

\section{Disclosure}

The authors report no conflicts of interest in this work.

\section{References}

1. Bernards R, Weinberg RA. A progression puzzle. Nature. 2002; 418(6900):823.

2. Koga T, Tokunaga E, Sumiyoshi Y, et al. Detection of circulating gastric cancer cells in peripheral blood using real time quantitative RT-PCR. Hepatogastroenterology. 2008;55(84):1131-1135.

3. He W, Wang H, Hartmann LC, et al. In vivo quantitation of rare circulating tumor cells by multiphoton intravital flow cytometry. Proc Nati Acad Sci U S A. 2007;104(28):11760-11765.

4. Gauthier LR, Granotier C, Soria JC, et al. Detection of circulating carcinoma cells by telomerase activity. Br J Cancer. 2001;84(5):631-635.

5. Devriese LA, Bosma AJ, van de Heuvel MM, et al. Circulating tumor cell detection in advanced non-small cell lung cancer patients by multimarker QPCR analysis. Lung Cancer. 2012;75(2):242-247.

6. SherYP, Shih JY, Yang PC, et al. Prognosis of non-small cell lung cancer patients by detecting circulating cancer cells in the peripheral blood with multiple marker genes. Clin Cancer Res. 2005;11(1):173-179.

7. Li Q, Qi H, Zhou HX, et al. Detection of micrometastases in peripheral blood of non-small cell lung cancer with a refined immunomagnetic nanoparticle enrichment assay. Int $J$ Nanomedicine. 2011;6: 2175-2181.

8. LuAH, SalabasEL, SchüthF. Magnetic nanoparticles: synthesis, protection, functionalization, and application. Angew Chem Int Ed Engl. 2007; 46(8):1222-1244.

9. Dilnawaz F, Singh A, Mohanty C,et al. Dual drug loaded superparamagnetic iron oxide nanoparticles for targeted cancer therapy. Biomaterials. 2010;31(13):3694-3706.

10. Jain TK, Morales MA, Sahoo SK, et al. Iron oxide nanoparticles for sustained delivery of anticancer agents. Mol Pharm. 2005;2(3):194-205.

11. Smith AM, Nie $\mathrm{S}$. Chemical analysis and cellular imaging with quantum dots. Analyst. 2004;129(8):672-677.

12. Goldman ER, Medintz IL, Mattoussi H. Luminescent quantum dots in immunoassays. Anal Bioanal Chem. 2006;384(3):560-563.

13. Pantel K, Brakenhoff RH. Dissecting the metastatic cascade. Nat Rev Cancer. 2004;4(6):448-456.

14. Mitas M, Hoover L, Silvestri G, et al. Lunx is a superior molecular marker for detection of non-small cell lung cancer in peripheral blood [corrected]. J Mol Diagn. 2003;5(4):237-242.

15. Betz C, Papadopoulos T, Buchwald J, et al. Surfactant protein gene expression in metastatic and micrometastatic pulmonary adenocarcinomas and other non-small cell lung carcinomas: detection by reverse transcriptase-polymerase chain reaction. Cancer Res. 1995; 55(19):4283-4286.
16. Dempo K, Satoh M, Tsuji S, et al. Immunohistochemical studies on the expression of pulmonary surfactant apoproteins in human lung carcinomas using monoclonal antibodies. Pathol Res Pract. 1987; 182(5):669-675.

17. Gerges N, Jabado N. Biomarkers in cancer micrometastasis: where are we at? Bioanalysis. 2010;2(5):881-899.

18. Massart R. Preparation of aqueous magnetic liquids in alkaline and acidic media. IEEE Trans Magn. 1981;17(2):1247-1248.

19. Lu SL, Forcada J. Preparation and characterization of magnetic polymeric composite particles by miniemulsion polymerization. $J$ Polym Sci Pol Chem. 2006;44(13):4187-4203.

20. Yan F, Li J, Zhang JJ, et al. Preparation of $\mathrm{Fe}_{3} \mathrm{O}_{4} /$ polystyrene composite particles from monolayer oleic acid modified $\mathrm{Fe}_{3} \mathrm{O}_{4}$ nanoparticles via miniemulsion polymerization. J Nanopart Res. 2009;11(2): 289-296.

21. Zhao K, Li J, Wang HZ, et al. Stoichiometric ratio dependent photoluminescence quantum yields of the thiol capping CdTe nanocrystals. J Phys Chem C. 2007;111(15):5618-5621.

22. Xu X, Friedman G, Humfeld KD, et al. Synthesis and utilization of monodisperse superparamagnetic colloidal particles for magnetically controllable photonic crystals. Chem Mater. 2002;14(3):1249-1256.

23. Wang PC, Chiu WY, Lee CF, et al. Synthesis of iron oxide/poly(methyl methacrylate) composite latex particles: Nucleation mechanism and morphology. J Polym Sci Pol Chem. 2004;42(22):5695-5705.

24. Sacanna S, Philipse AP. Preparation and properties of monodisperse latex spheres with controlled magnetic moment for field-induced colloidal crystallization and (dipolar) chain formation. Langmuir. 2006;22(24):10209-10216.

25. Deng Y, Wang L, Yang W, et al . Preparation of magnetic polymeric particles via inverse microemulsion polymerization process. J Magn Magn Mater. 2003;257(1):69-78.

26. Ramírez LP, Landfester K. Magnetic polystyrene nanoparticles with a high magnetite content obtained by miniemulsion processes. Macromol Chem Phys. 2003;204(1):22-31.

27. Wang Y, Teng XW, Wang JS, et al. Solvent-free atom transfer radical polymerization in the synthesis of $\mathrm{Fe}_{2} \mathrm{O}_{3} @$ polystyrene core-shell nanoparticles. Nano Lett. 2003;3(6):789-793.

28. Vestal CR, Zhang Z. Atom transfer radical polymerization synthesis and magnetic characterization of $\mathrm{MnFe}_{2} \mathrm{O}_{4}$ /polystyrene core/shell nanoparticles. J Am Chem Soc. 2002;124(48):14312-14313.

29. Michalet X, Pinaud FF, Bentolila LA, et al. Quantum dots for live cells, in vivo imaging, and diagnostics. Science. 2005;307(5709):538-544.

30. Gao X, Cui Y, Levenson RM, et al. In vivo cancer targeting and imaging with semiconductor quantum dots. Nat Biotechnol. 2004; 22(8):969-976.
International Journal of Nanomedicine

\section{Publish your work in this journal}

The International Journal of Nanomedicine is an international, peerreviewed journal focusing on the application of nanotechnology in diagnostics, therapeutics, and drug delivery systems throughout the biomedical field. This journal is indexed on PubMed Central, MedLine, CAS, SciSearch $\AA$, Current Contents ${ }^{\circledR} /$ Clinical Medicine,

\section{Dovepress}

Journal Citation Reports/Science Edition, EMBase, Scopus and the Elsevier Bibliographic databases. The manuscript management system is completely online and includes a very quick and fair peer-review system, which is all easy to use. Visit http://www.dovepress.com/ testimonials.php to read real quotes from published authors. 